Circle of scientific interests: theory rings, theory and methodology for teaching mathematics.

OVSIANIK Tetiana Sergiivna - math teacher of I-III levels school of Velykoandrusivka village's council
Circle of scientific interests: competency approach to teaching mathematics at school.

Стаття надійшла до редакиії 21.09.2020 p
УДК 373.5.091.39:81'241

DOI: 10.36550/2415-7988-2020-1-191-208-212
АНДРЕСВ Антон Геннадійович аспірант 3 року навчання кафедри німецької мови і літератури з методикою викладання Криворізького державного педагогічного університету ORCID:https://orcid.org/0000-0003-2457-8918 e-mail: mjawets@gmail.com

\title{
PRÄVENTION VON KONFLIKTEN UND RASSISMUS AUF NATIONALER EBENE UNTER OBERSCHÜLERN
}

Erklärung und begründung der dringlichkeit des problems . Wir leben in einer dynamischen zeit, wo fast jeder, der diesen wunsch hat, in ein anderes land auswandern kann. Dazu können verschiedene motive (studium, arbeit, urlaub) führen. Deshalb entstand in den letzten jahrzehnten in vielen ländern europas, die multikulturelle züge aufweisen, das problem der sozialisierung von kindern, deren eltern arbeitsmigranten sind. Eine der wichtigsten sphären, die sich für sozialisation am besten eignet, ist die schule. Dieses thema ist nicht neu, aber es verliert nicht an aktualität.

Analyse aktueller forschungen und veröffentlichtungen. Die meisten Schriften und Bücher, die das Thema „Prävention des Rassismus“ und „Interkulturelle Konflikte" zum Gegenstand der Forschung gemacht haben, interpretieren die obengenannten Probleme als „,interkulturelle Missverständnisse“ (Bundesprogramm „Demokratie Leben“ [13], Netzwerk Projekt „Schule ohne Rassismus - Schule mit Courage" [14]) und berücksichtigen selten die psychologische Seite des Problems oder lassen sie außer Acht. Die Konflikte in einer heterogenen Gesellschaft entstehen öfter deswegen, dass man psychologische Probleme hat; interkulturelle Missverständnisse können auch zu Konflikten führen, aber das kommt seltener vor, wenn persönliche Gründe für den Hass dem Anderen gegenüber fehlen.

Ziel des artikels. Das Ziel des Artikels besteht darin, das Problem vom Wesen und von Motiven der Konflikte auf dem nationalen Grund und Rassismus unter Schülern als eine soziale Erscheinung aus psychologischer Sicht $\mathrm{zu}$ erforschen und mögliche Präventionsmaßnamen anzubieten.

Forschungsmethoden.

Die Grundforschungsmethoden, die in diesem Artikel eingesetzt wurden, sind: theoretische Analyse (die Erforschung der grundlegenden theoretischer Begriffe; die Analyse der wissenschaftlichen Literatur zum Forschungsthema); kritische Analyse (die Statusfrage von Rassismus unter Oberschülern als psychologisches Problems; die Gründe für rassistische Ansichten bei den Schülern; Vergleichende Charakteristika von Schulrassisten, Opfern und Bullys); Systemanalyse (die Auswahl des faktischen Materials und ihre Gruppierung);
Beschreibungsmethode (die Beschreibung von eventuellen Präventions-maßnamen des Schulrassismus).

Präsentation des hauptmaterials der forschung. Als Erstes, betrachten wir den Begriff «Rassismus» und sein Wesen. Unter dem Begriff «Rassismus» versteht man eine Lehre oder eine Theorie, nach der die Menschen bzw. Bevölkerungsgruppen mit bestimmten biologischen Merkmalen hinsichtlich ihrer kulturellen Leistungs-Fähigkeit anderen von Natur aus über- bzw. unterlegen erscheinen. Man unterscheidet verschiedene Formen des Rassismus - darunter den sogenannten «reaktiven Rassismus».

Th. Teo bezeichnet diese Erscheinung als «reaktiven Rassismus», weil in ihrem Hintergrund die Reaktion auf das rassistische Verhältnis steht [16]. Voraussetzungen für solche Ansichten stammen aus den 30-er Jahren des 20. Jahrhunderts. Ursprünglich basierten sie auf der Idee, dass die Menschen mit schwarzer Hautfarbe obergestellt sind. und entstanden wegen Tätigkeit «Nation of Islam» (ein politische und religiöse Organisation). Die berühmtesten Vertreter dieser Bewegung waren Muhammad Ali (Cassius Clay) und Malcolm X (Malcolm Little) [6, S. 100-103]. Ungeachtet der Tatsache, dass heute der Multikulturalismus zum Trend geworden ist, verschwindet solche Erscheinung wie Rassismus nicht; sogar umgekehrt: sie umfasst alle Lebensbereiche, aber oft ist sie verschleiert und nicht so offen demonstriert.

Die Schule ist ein kleines Modell der Gesellschaft, und, wie jede Gesellschaft, hat sie auch ihre eigene Hierarchie, Probleme und Konflikte unter ihren Mitgliedern, einschließlich national bedingter Konflikte auf ethnischem Grund.

Bevor wir die Ursachen von Teenagerrassismus analysieren, wäre es sinnvoll, den Charakter eines durchschnittlichen Oberschülers, seinen psychologischen Zustand und die Besonderheiten von seinem Alter zu betrachten. Bei dieser Untersuchung hilft uns die Forschung von Hilko M. und Tkatscheva M. [8], wo Psychologie des Menschen altersgemäß analysiert wird. Laut ihrer Forschung ist ein Oberschüler:

1) eine Person von 15-17 Jahren in der Ukraine, 1518 Jahren in den USA, 15-19 Jahren in Deutschland; 
2) er verfügt über solche Eigenschaften, wie: Instabilität des Nervensystems, Erregbarkeit, breite Emotionspalette usw.;

3) ist abhängig von der Meinung der nächsten Umgebung [8, S. 103-105].

Die Person in diesem Alter beginnt öfter und tiefer die Umwelt zu reflektieren, denkt mehr über den eigenen psychologischen Zustand und analysiert ihn (obwohl oft nicht objektiv), bildet das eigene Persönlichkeitsideal, empfindet das Bedürfnis nach vertraulicher Kommunikation, neigt zu verschiedenen Protestformen und hat einen Wunsch, sich von den anderen zu unterscheiden und gleichzeitig NICHT zu unterscheiden. In einem der Werke von Hilko und Tkatscheva «Vozrastnaja psihologia»[8], bemerken Autoren, dass Teenager verschiedene Eigenschaften im kognitiven Bereich haben, und zwar:

- Jungen neigen zu einer abstrakten Denkweise;

- Mädchen - zu einer konkreten;

- ihre Aufmerksamkeit ist selektiv;

- sie neigen zur Übertreibung ihrer eigenen Einzigartigkeit;

- ihre Autoritäten werden oft geändert, manchmal verlieren sie an Bedeutung;

- für sie ist ihr eigenes Aussehen und das Aussehen der Anderen sehr wichtig;

- sie suchen oft nach ihren eigenen Mängeln und auf ihrem Grund können sich verschiedene Komplexe entwickeln;

- oft haben sie ein Bedürfnis nach einem geistigen Mentor, aber nicht in der Gestalt von Eltern oder Lehrern, sondern eines «älteren Freundes»;

- nicht selten kommt die Entfremdung von der üblichen Umgebung vor;

- sie leiden wegen der Unmöglichkeit, die eigene Persönlichkeit und ihre eigene innere Welt zu demonstrieren, weil die Gestalt vom «Ich-Konzept» unklar, undeutlich oder unvollendet ist [8, S. 20-55].

Mit Rücksicht darauf, was oben dargelegt wurde, kann man behaupten, dass jeder Schüler eine Art „Pulverfass“ ist, das akut auf alles Mögliche und Unmögliche reagiert, aber gleichzeitig sich leicht beeinflussen lässt. Deswegen ist es kein Wunder, dass sogar in einer Schulgesellschaft rassistische Stimmungen erscheinen können.

Was sind die Gründe für rassistische Ansichten bei Schülern?

Man kann sie in 3 Gruppen unterteilen:

1) persönliche (eigene negative Erfahrung);

2) interkulturelle (z.B. verschiedene geopolitische Konflikte, die eine vorgefasste Meinung (Vorurteile) über etwas oder jemanden bilden);

3) äußere (externe) (negative Erfahrung von den Eltern, Freunden, Bekannten; Propaganda).

Alle obengenannten Gründe kann man am Beispiel von Ereignissen in Großbritannien am Ende der 60-er Jahre des 20. Jahrhunderts beobachten. In dieser Zeit kam eine große Gruppe von Migranten aus Pakistan nach Großbritannien. Konservative Engländer und Pakistaner mit ihren Vorurteilen haben ihre eigene negative Erfahrung auf ihre Kinder übertragen. Wegen der
Tätigkeit von «Nationaler Front» (Nationale Front/die Britishe Nationale Front - ist eine radikalenationalistische politische Partei) wurde mit Hilfe von Propaganda die Feindschaft nicht nur unter Erwachsenen, sondern auch innerhalb der heranwachsenden Generationen entfacht. Als Folge von dieser Feindschaft nennt man bis heute Pakistaner «Paki», ungeachtet der Tatsache, dass 3 von 4 Pakistanern in Großbritannien geboren wurden oder Kinder in internationalen Ehen sind.

Erinnern wir uns an Russland und seine Tschetschenischen Kriege. Der erste Krieg dauerte von 1994 bis 1996 und der zweite von 1999 bis 2009. Als Folge entstanden in Russland viele rechtsradikale Bewegungen. Und bis zum Jahr 2013 gehörten nationalistisch gefärbte Konflikte in verschiedenen Formen zum Alltag jeder großen Stadt. So kann man behaupten, dass zwei Kriege zur Feindschaft unter Vertretern von zwei ethnischen Gruppen im Rahmen eines Landes im Zeitraum von zwei Generationen geführt haben.

«Dank» solchen Ereignissen wie Kriege und wirtschaftliche Krisen entsteht eine für den Durchbruch von Rechts- und rassistischer Propaganda günstige Atmosphäre, die einen großen Einfluss auf die Jugendlichen ausübt und ihre unreife Vernunft gewinnt.

Die Wissenschaftler P. Pinaev und J. Jatskevitsch definieren den radikalen Nationalismus als eine Äußerung der Neigung einer Person zur Gewalt [12]. Das gleiche kann man auch über den Rassismus sagen.

Im Fall, wenn ein Schüler keines von den obengenannten Motiven hat, geht es höchstwahrscheinlich nicht um Rassismus, sondern einfach um Aggression.

Mit Rücksicht auf das oben Gesagte, kann ein Rassist wie folgt betrachtet werden:

Wir alle wissen, dass es in jeder Schule oder sogar in jeder Klasse einen oder mehrere sogenannte Aggressoren gibt. Basierend auf der These von P. Pinaev und J. Jatskevitsch [12], kann man behaupten, dass die Verhaltensmuster von einem einfachen Schul-Aggressor und von einem Schul-Rassisten in der Konfliktsituation zusammenfallen. Der einzige Unterschied ist das Objekt ihrer Aggressivität.

Dies führt uns zur Idee, dass der echte Aggressionsgrund im psychologischen Zustand des Schülers verankert ist.

Es wurde festgestellt, dass nur $25 \%$ von Konflikten in der Schule aufgrund des Rassismus und der Religion möglich sind und aufgrund der nationalen oder ethnischen Zugehörigkeit nur $8 \%$. Die Wissenschaftler aus Dänemark haben direkten Zusammenhang zwischen der Körperstrafe in der Familie und dem Bullying entdeckt. A. Korol in ihrem Artikel über die Ursachen und Konsequenzen vom Bullying [6] sagt, dass die gleiche Meinung amerikanische Wissenschaftler (N. Meyer-Adams, B. T. Conner) haben, die bemerkten, dass die Jungen, die „Buller“ sind, oft als Opfer von Familiengewalt auftreten. Britische Wissenschaftler (D. Pepler, K. Rigby) betonten, dass die emotionale Ablehnung von Eltern ihrer Kinder oft zum devianten 
Benehmen von Teenagern führen kann. In der Tabelle 1 sind wesentliche Charakteristika von Schulrassisten, Opfern und Bullys zusammengefasst, die den Vergleich dieser Schülerkategorien mitteinander ermöglichen. Diese Tabelle wurde aufgrund von Werken N. MeyerAdams, B. T. Conner, K. Rigby, A. Korol, P. Pinaev, J. Jatskevtsich, A. Borschtschevska entwickelt. Hier muss man betonen, dass diese Tabelle nur die Angaben für den Fall der körperlichen Gewalt wiederspiegelt.

Tabelle 1.

Vergleichende Charakteristika von Schulrassisten, Opfern und Bullys

\begin{tabular}{|c|c|c|}
\hline \multicolumn{3}{|c|}{ Opfern und Bullys } \\
\hline Schulrassist & Opfer & Bullys \\
\hline $\begin{array}{l}\text { Probleme in der } \\
\text { Familie }\end{array}$ & $\begin{array}{l}\text { Probleme in } \\
\text { der Familie }\end{array}$ & $\begin{array}{l}\text { Probleme in der } \\
\text { Familie }\end{array}$ \\
\hline Familiengewalt & $\begin{array}{c}\text { Familiengewal } \\
\mathrm{t}\end{array}$ & Familiengewalt \\
\hline $\begin{array}{l}\text { keine Familien- } \\
\text { unterstützung }\end{array}$ & $\begin{array}{c}\text { keine } \\
\text { Familien- } \\
\text { unterstützung }\end{array}$ & $\begin{array}{c}\text { keine } \\
\text { Familienunterst } \\
\text { ützung }\end{array}$ \\
\hline $\begin{array}{c}\text { unbewusste } \\
\text { Rache }\end{array}$ & $\begin{array}{l}\text { unbewusste } \\
\text { Rache }\end{array}$ & $\begin{array}{l}\text { unbewusste } \\
\text { Rache }\end{array}$ \\
\hline $\begin{array}{l}\text { ziellose } \\
\text { Existenz }\end{array}$ & $\begin{array}{c}\text { ziellose } \\
\text { Existenz }\end{array}$ & $\begin{array}{l}\text { ziellose } \\
\text { Existenz }\end{array}$ \\
\hline $\begin{array}{c}\text { niedrige } \\
\text { Selbstachtung, } \\
\text { Selbstzweifel }\end{array}$ & $\begin{array}{c}\text { niedrige } \\
\text { Selbstachtung } \\
\text { Selbstzweifel }\end{array}$ & $\begin{array}{c}\text { niedrige } \\
\text { Selbstachtung } \\
\text { Selbstzweifel }\end{array}$ \\
\hline Aggressivität & - & Aggressivität \\
\hline nicht sozialisiert & $\begin{array}{c}\text { nicht } \\
\text { sozialisiert }\end{array}$ & nicht sozialisiert \\
\hline
\end{tabular}

Eigene Quelle

Wenn man diese Tabelle aufmerksam studiert, dann kann man verstehen, dass diese 3 Typen in ihren Charakteristika im Großen und Ganzen zusammenfallen. Den einzigen Unterschied bildet die Reaktion. Im Fall «Bully» und «Schulnazi» transformieren die Schüler ihre Ängste in die Aggression und übertragen sie auf andere Kinder. Wegen Besonderheiten des Charakters wie, z.B., Gutherzigkeit, ausgeglichenes Temperament u.a. werden Schüler mit gleichen Familienproblemen als «Opfer» bezeichnet, weil sie ihre Ängste nicht transformieren können. Aber es ist wichtig zu betonen, dass wir in diesem Fall nur über körperliche Gewalt sprechen, weil im psychologischen und sozialen Bereich solch ein Benehmensmodell für Oberschüler nicht normal ist. Deshalb fordern «Opfer», die mit Gewalt einverstanden sind, „Schul-Nazi“ und Bully, die diese Gewalt ausüben, gleiche Menge von Aufmerksamkeit, weil körperliche Gewalt nur für die Kinder aus dysfunktionalen Familien zur Normalität gehört. Diejenigen, die andere «BullyingArten» wie «verbale» oder «cýber» Bulling praktizieren, stammen oft aus ganz normalen Familien, so wie auch ihre «Opfer», und am meisten streben sie nach Dominanz und Macht [3, S. 101-104]. Im Vergleich zu einem Bully, der alle Probleme mit Hilfe von Fäusten löst und asozial ist, kann man einen «verbalen» Bully als einen «sozialisierten» Typ betrachten. Dieser hat oft gute Beziehungen mit Kommilitonen, bessere Noten (als die Asozialen) und die Lehrer nehmen ihn auch am meisten nicht als einen Bully wahr [9]. Deshalb ist das deviante Benehmen in diesem Fall nichts Anderes als die eigene Angst, die man, um sich zu wehren und zu sichern, in die Aggression den Anderen gegenüber transformiert und die man später auf andere Kinder überträgt. Nicht selten treten die Kinder, die bestimme Probleme in der Familie haben, verschiedenen Organisationen und Gruppierungen bei, entweder nationalistischen, oder auch rassistischen, weil sie nur dort die fehlende Unterstützung und Achtung finden und die Wichtigkeit ihrer eigenen Persönlichkeit wahrnehmen.

Jetzt übergehen wir unmittelbar zu den eventuellen Präventionsmaßnamen.

1) In erster Linie muss man als Pädagoge regelmäßig und fachlich den psychologischen Zustand von Schülern einschätzen, um vorher die Individuen zu bestimmen, die zum devianten Benehmen und $\mathrm{zu}$ Aggressionsanfällen neigen, und deren Situation in der Familie näher studieren.

Die Schüler, die sich als potentiell aggressiv aufweisen, kann man zu den speziellen Maßnahmen heranziehen, die ihre Aggressivität in eine andere Richtung lenken, z.B. das können allerartige außerschulische Sportgruppen sein, wo die negativen Emotionen und ihre Energie in einen Wettbewerbsgeist und Gewinnwillen transformiert werden kann [5].

2) Ganz wichtig ist es, im Voraus zu adäquaten und positiven Einstellungen $\mathrm{zu}$ anderen Nationen und Völkern zu erziehen. Außer allermöglichem Informieren über fremde Kulturen und fremdes Alltagsleben, wäre es zweckmäßig, die Zusammenarbeit von Schülern, die zu verschiedenen Nationen / Völkern gehören, zu gewährleisten und darauf $\mathrm{zu}$ achten, dass die Schülergemeinschaft nicht in «nationale Gruppen» zerfällt. Sehr produktiv erweist sich die gemeinsame Arbeit der «gemischten» Gruppen in Schulprojekten, wo die Schüler miteinander eng zusammenwirken können und ihre Tätigkeit miteinander koordinieren müssen. Ähnliche Praxis nutzte das «Centrum für angewandte Politikforschung CAP der Ludwig-MaximiliansUniversität München» in seiner Programm «Kinder gegen Rassismus - Kinder für Toleranz».

3) Im Fall, wenn die Konflikte unter den Schülern schon entstanden sind und (am wichtigsten!) sich genau als Konflikte auf dem nationalen Grund bestätigt haben, muss man zu bestimmten Extra-Maßnahmen greifen. Privatgespräch mit dem Schüler wäre in diesem Kontext am hilfreichsten, und zwar erfolgt es nach diesem Schema:

- $\quad$ die Motive der Tat feststellen;

- die Eltern dieses Schülers in Kenntnis

setzen;

wenn die Motive keinen persönlichen Charakter tragen, kann man den Schüler in die logische Verlegenheit bringen, damit er das Problem selbst versteht, dass er seine Handlungen auf keine Weise begründen kann;

- $\quad$ erklären, dass die Liebe zu eigenem Volk, zu seiner Nationalität, Kultur usw. ganz normal ist.

Dies gibt aber Keinem das Recht, die anderen wegen ihrer Nationalität zu erniedrigen. Man kann 
berühmte Beispiele anführen, wie: Muhammad Ali (der berühmte Boxer), Malcolm X, oder Bryon Widner. Diese Leute waren echte Rechtsradikale, aber sie haben rechtzeitig verstanden, dass nicht alle Leute gleich sind und die Gewalt immer nicht zur Lösung des Problems, sondern zur noch größeren Gewalt führt.

4) Im Fall, wenn die Konflikte unter den Schülern den höchsten Konfrontationspunkt erreicht haben, und zwar psychische oder physische Gewalt, Verfolgung usw. auf dem nationalen Grund als Folge haben, könnte die letzte Maßnahme eine Exkursion in die Haftanstalten sein.

Es ist besser für die Schüler sich selbst zu überzeugen, welche Konsequenzen ihr deviantes oder, wie es in ihren Augen aussieht, radikales Benehmen haben kann, als 1000 mal das von den Eltern oder Lehrern zu hören, die übrigens oft keine Autorität für Kinder sind. Ähnliche Methodik wurde in TV-Show Beyond Scared Straigth (ein Bestandteil von YIPA (Youth Intervention Programm) Programm, das sich mit Umerziehung von den Schwierigen Kindern beschäftigt) ausprobiert. Solche Praxis fand in Norwegen «Ullersmo Project», Australien «Day in gaol», Großbritannien «Day visits» statt. [4]. Die Kinder müssen am Beispiel der Anderen sehen und akzeptieren, dass der Rassismus keine Weltanschauung ist, sondern in erster Linie mit der Verletzung der Menschenrechte verbunden ist und als ein schweres Verbrechen von der Gesellschaft definiert wird. Und das Wichtigste ist, dass dies kein Verbrechen gegen Schwarz / Weiß / Gelb, sondern ein Verbrechen gegen MENSCHEN ist.

Schlussfolgerungen aus der studie und perspektiven für die weitere entwicklung. Zusammenfassend kann man sagen, dass der Rassismus in erster Linie kein interkulturelles, sondern ein psychologisches Problem ist. Deswegen dürfen wir als Pädagogen dem Schüler eine fremde Kultur aufzwingen, sondern ihm helfen, mit sich selbst zurechtzukommen.

Für Rassismus und Nationalismus gibt es immer Ursachen. Und zwar persönliche, interkulturelle und andere. Oberschüler können wegen ihrer psychologischen Altersbesonderheiten auf alle äußeren Reizfaktoren sehr emotional reagieren, aber auf jeden Fall ist die physische Gewalt nicht normal für sie. Wie auch nicht normal ist für einen Oberschüler in gesunder Psyche und gesundem Familienklima, zum Opfer physischer Gewalt zu werden.

Man kann aber eine Reihe von Präventionsmaßnahmen gegen Rassismus den Oberschülern anbieten. In erster Linie wäre es nützlich den psychologischen Zustand von Schülern zu untersuchen. Nach der Entdeckung von potentiell aggressiven Schülern könnte man ihre negative Energie in eine andere Richtung lenken, z.B. verschiedene Sportund Freizeitaktivitäten anbieten. Wie schon festgestellt wurde, kommen solche Schüler aus den dysfunktionalen Familien, und gerade in solchen Gruppen haben sie die Möglichkeit, fehlende Achtung und Unterstützung zu bekommen.

Die zweite mögliche Präventionsmaßnahme wäre die Zusammenarbeit im Unterricht (oder auch in der
Arbeit außerhalb des Unterrichts) der Schüler, die zu verschiedenen Nationen oder Kulturen gehören. Unter den Bedingungen, wenn die Schüler unbedingt kooperieren müssen, erfolgt der Prozess des gegenseitigen Verständnisses viel schneller als in einem normalen Unterricht.

Im Fall, wenn die Konflikte unter den Schülern schon entstanden sind und sich genau als Konflikte auf dem nationalen Grund bestätigt haben, wären zwei mögliche Maßnahmen zweckmäßig: ein Privatgespräch führen und / oder das negative Beispiel anführen.

Das erste erweist sich als effektiv, wenn die Schüler sich von Erwachsenen (und zwar von den Eltern oder vom Lehrer) mehr oder weniger beeinflussen lassen. Doch wie schon oben erwähnt ist, sind die Erwachsenen aus der Umgebung der Schüler oft kaum eine Autorität für sie. Dann wäre das reale Beispiel sehr hilfreich: aus der ersten Quelle hört der Schüler, was passieren kann, wenn man sich in dieser Richtung weiterbewegt.

Natürlich müssen diese Maßnahmen in der Praxis noch konkret überprüft werden, aber, wenn man die Psychologie der Schüler in Kauf nimmt und von diesem Standpunkt aus die Situation einschätzt, kann man vermuten, dass sie sich höchstwahrscheinlich als effektiv erweisen.

\section{LITERATUR}

1. Adams-Meyer N., Conner T. B.: School Violence: Bullying Behaviors and the Psychosocial School Environment in Middle Schools Children \& Schools, Volume 30, Issue 4, 2008. $211 \mathrm{~S}$.

2. Borschtschevska A. Bulling u schkolah jak tschinnyk psychitschnoho nezdorovja. Naukovyi vistnyk Mizhnarodnogo gumanitarnogo universytetu №6. Serie «Medizin», 2014. S. 4852.

3. Coloroso B.: The Bully, the Bullied, and the Bystander. New York: William Morrow Paperbacks, 2009. 246 S.

4. Beyond Scared Straight's Real-Life Controversy: Daily Beast Magazine. URL: https://www.thedailybeast.com/beyond-scared-straights-reallife-controversy (Aufrufdatum: 22.08.2020).

5. Ehninger F., Perlich M., Schuster K.-D.: Streitschlichtung und Umgang mit Gewalt an Schulen. Magdeburg: Fridrich-Ebert-Stiftung Landesbüro SachsenAnhalt, 2007. $88 \mathrm{~S}$

6. Gibson D.-M.: A History of the Nation of Islam : Race, Iclam, and the Quest for Freedom. Praeger, 2012. 240 S.

7. Hill Collins P.: From Black Power to Hip Hop: Racism, Nationalism, and Feminism. United States of America, Philadelphia: Temple University Press, 2006. 248 S.

8. Hilko M., Tkatscheva M.: Vozrastnaja psichologia. Moskva: Jurajt, 2014. $201 \mathrm{~S}$.

9. Kolesnikova, L.: Batkivske nasillja ta jogo naslidky jak problema simejnogo vyhovannja u Nimetschyni, 2009. URL:http://ea.donntu.edu.ua/bitstream/123456789/11180/4/кол есникова.pdf (Aufrufdatum: 29.08.2020).

10. Korol A.: Prytschyny ta naslidky javyschtscha bullingu. Kyjiw: Vidnovne pravosuddja v Ukraini № 1-2, 2009. S. 84-93.

11. Miroshnikova, V.: Tschernyj rasizm $\mathrm{v}$ sotsialnoekonomitscheskom aspekte SSCHA v XXI veke, 2017. URL: https://cyberleninka.ru/article/n/chernyy-rasizm-v-sotsialnoekonomicheskom-aspekte-ssha-v-xxi-veke 20.08.2020). 
12. Pinaev P., Jatskevitsch J.: Natsionalizm v molodyozhnoj srede sovremennogo rossijskogo obschtschestva. Vestnik Permskogo Universiteta №3. Serie «Philosophie. Psychologie. Soziologie», 2014. S. 137-147.

13. Projekte zur Prävention von Rassismus und rassistischer Diskriminierung im Bundesprogramm «Demokratie leben!». URL: https://www.bmfsfj.de/blob/ 130892/ 7230ab6286c1784b3abb79ace884d/projekte-zurpraevention-von-rassismus-und-rassistischerdikriminierugdata.pdf. (Aufrufdatum: 25.08.2020).

14. Schule ohne Rassismus: Netzwerk. URL: https://m.schule-ohne-rassismus.org/startseite/ (Aufrufdatum: 25.08.2020).

15. Smith P.K., Pepler D., Rigby K.: Bulling in schools: How successful can interventions be? Cambridge, United Kingdom: Cambridge University Press, 2004. 334 S.

16. Teo T.: Rassismus: eine psychologisch relevante Begriffsanalyse, $1995 . \quad$ URL: https://www.ssoar.info/bitstream/handle/document/2963/ssoarjourn psycho-1995-3-teorassismus_eine_psychologisch_relevante_begriffsanalyze.pdf (Aufrufdatum: 11.09.2020).

\section{REFERENSES}

1. Adams-Meyer, N., Conner, T. B., (2008). School Violence.

2. Borschtschevska, A., (2014). Bulling u schkolah jak tschinnyk psychitschnoho nezdorovja [Bulling in schools as a factor of a mental illness].

3. Coloroso, B. (2009). The Bully, the Bullied, and the Bystander. New York.

4. Beyond Scared Straight's Real-Life Controversy.

5. Ehninger, F., Perlich, M., Schuster, K.-D., (2007). Streitschlichtung und Umgang mit Gewalt an Schulen [Dispute settlement and dealing with violence in schools]. Magdeburg.

6. Gibson, D.-M., (2012). A History of the Nation of Islam.

7. Collins P., Hill., (2006). From Black Power to Hip Hop: Racism, Nationalism, and Feminism. Philadelphia.

8. Hilko, M., Tkatscheva, M., (2014): Vozrastnaja psichologia [Age-related psychology]. Moscow.

9. Kolesnikova, L., (2009). Batkivske nasillja ta jogo naslidky jak problema simejnogo vyhovannja u Nimetschyni
[Domestic violence and its consequences as a problem of family upbringing in Germany].

10. Korol, A., (2009). Prytschyny ta naslidky javyschtscha bullingu [Causes and consequences of the bullying phenomena]. Kyiv.

11. Miroshnikova, V., (2017). Tschernyj rasizm $v$ sotsialno-ekonomitscheskom aspekte SSCHA v XXI veke [Black racism in the socio-economic aspect of the United States of America in the 21st century].

12. Pinaev, P., Jatskevitsch, J., (2014). Natsionalizm v molodyozhnoj srede sovremennogo rossijskogo obschtschestva [Nationalism among the youth of modern Russian society]. Perm.

13. Projekte zur Prävention von Rassismus und rassistischer Diskriminierung im Bundesprogramm «Demokratie leben!» [Projects for prevention of racism and racial discriminations in the federal program «Live democraty»].

14. Schule ohne Rassismus: Netzwerk [School without racism: network].

15. Smith, P.K., Pepler, D., Rigby, K., (2004). Bulling in schools: How successful can interventions be? Cambridge.

16. Teo, T., (1995). Rassismus: eine psychologisch relevante Begriffsanalyse [Racism: a psychologically relevant concept analysis].

\section{ВІДОМОСТІ ПРО АВТОРА}

АНДРЕЄВ Антон Геннадійович - аспірант 3 року навчання кафедри німецької мови і літератури з методикою викладання Криворізького державного педагогічного університету.

Наукові інтереси: проблема виховання учнів в мультикультурному суспільстві.

\section{INFORMATION ABOUT THE AUTHOR}

ANDREIEV Anton Hennadiyovych - $\mathrm{PhD}$ candidate of the third year of study of the Department of the German Language and Literature with Methods of Teaching of Kryvyi Rih State Pedagogical University.

Circle of research interests: the problem of the education of students in a multicultural society.

Стаття надійшла до редакиіï 22.09.2020 p.

\section{УДК 37.02}

DOI: 10.36550/2415-7988-2020-1-191-212-216

БЕВЗ Анна Володимирівна аспірантка кафедри природничих наук та методик їх викладання Центральноукраїнського державного педагогічного університету імені Володимира Винниченка ORCID:https://orcid.org/0000-0001-8989-5784 e-mail: annabevz.kr.ua@gmail.com

\section{ОСОБЛИВОСТІ ФОРМУВАННЯ ПРОФЕСІЙНОЇ КОМПЕТЕНТНОСТІ ФАХОВИХ МОЛОДШИХ БАКАЛАВРІВ}

Постановка та обгрунтування актуальності проблеми. Сучасний оцифрований світ розвивається інтенсивним шляхом. Промисловість, сільськогосподарське виробництво, наука, культура та інші галузі стають все більш глобальними, що призводить до високої конкуренції на ринку праці. На сьогодні для роботодавців найбільш привабливими $\epsilon$ ті випускники, що володіють сукупністю теоретичних знань, професійних умінь і навичок, які здатні вирішувати професійні проблеми та завдання в реальних ситуаціях 3 використання знань та життєвого досвіду [2]. За таких умов конкурентоспроможними можуть бути фахівці, що швидко, гнучко і креативно мислять, оперативно приймають зважені рішення для розв'язання 